Swiss Finance Institute

Research Paper Series N¹3-43

\title{
Can CRRA Preferences Explain CAPM-Anomalies in the Cross-Section of Stock Returns?
}

Sabine ELMIGER

University of Zurich and Swiss Finance Institute (PhD Program) 
Established at the initiative of the Swiss Bankers' Association, the Swiss Finance Institute is a private foundation funded by the Swiss banks and Swiss Stock Exchange. It merges 3 existing foundations: the International Center FAME, the Swiss Banking School and the Stiftung "Banking and Finance" in Zurich. With its university partners, the Swiss Finance Institute pursues the objective of forming a competence center in banking and finance commensurate to the importance of the Swiss financial center. It will be active in research, doctoral training and executive education while also proposing activities fostering interactions between academia and the industry. The Swiss Finance Institute supports and promotes promising research projects in selected subject areas. It develops its activity in complete symbiosis with the NCCR FinRisk.

The National Centre of Competence in Research "Financial Valuation and Risk Management" (FinRisk) was launched in 2001 by the Swiss National Science Foundation (SNSF). FinRisk constitutes an academic forum that fosters cutting-edge finance research, education of highly qualified finance specialists at the doctoral level and knowledge transfer between finance academics and practitioners. It is managed from the University of Zurich and includes various academic institutions from Geneva, Lausanne, Lugano, St.Gallen and Zurich. For more information see www.nccr-finrisk.ch .

This paper can be downloaded without charge from the Swiss Finance Institute Research Paper Series hosted on the Social Science Research Network electronic library at:

http://ssrn.com/abstract=2312273 


\title{
Can CRRA Preferences Explain CAPM-Anomalies in the Cross-Section of Stock Returns?
}

\author{
Sabine Elmiger*
}

August 5, 2013

\begin{abstract}
A large number of empirical studies find evidence for systematic deviations from the CAPM. The CAPM tends to understate the returns on low-beta stocks and overstate the returns on high-beta stocks, which means that the security market line is too steep. Other well-documented anomalies are the size premium and the value premium. The CAPM is a special case of the consumption-based CAPM. This study adresses the question whether the consumption-based CAPM with constant relative risk aversion preferences can explain CAPM-anomalies. An example of an economy with power utility and lognormal returns is examined that can be solved in closed form. The model leads to a security market line that is flatter than in the CAPM and generates a size and a value premium. The comparative statics suggest that cross-sectional anomalies and the equity premium puzzle are of a very similar nature.
\end{abstract}

Keywords: CAPM, CCAPM, CRRA, lognormality, multiple assets, beta premium, value premium, size premium

\section{Introduction}

The traditional capital asset pricing model (CAPM) of Sharpe (1964), Lintner (1965) and Mossin (1966) is one of the cornerstones of finance. It provides a simple explanation about what type of risk drives the cross-section of asset returns and how to measure it. More precisely, it states that expected asset returns are solely determined by the assets' exposure to market risk.

\footnotetext{
${ }^{\sqrt{3}}$ This is part of my PhD thesis. I would like to thank my supervisor Thorsten Hens for helpful comments and discussions. I am grateful to the Hausdorff Institute for Mathematics in Bonn, where I completed this work in summer 2013.

${ }^{*}$ SFI-Ph.D. student at the University of Zurich, Plattenstrasse 32, CH-8032 Zurich, sabine.elmiger@bf.uzh.ch
} 
The measure for this is the so-called beta factor, which measures the covariance between the assets' return with the return on the market portfolio scaled by the variance of the market return. The CAPM is a very elegant pricing model, but its empirical validity is highly debated.

The CAPM says that expected excess returns should be proportional to their beta with a factor of proportionality equal to the expected market excess return. Different empirical studies show that there is indeed a linear relationship between assets' expected excess returns and their betas, but the line is too flat. Excess returns on low-beta assets tend to be too high, and excess returns on high-beta assets tend to be too low. For a discussion of this observation see Frazzini and Pedersen (2011) and the survey of Fama and French (2004) and references therein.

In the empirical literature, there is evidence that many different sorting procedures other than beta-sorting lead to significant deviations from the CAPM. The most prominent examples of this evidence are probably the size and the value premium. Banz (1981) finds that stocks with a small market capitalization have higher expected returns than the CAPM predicts, and Rosenberg et al. (1985) report that stocks with a high book-to-market ratio (value stocks) tend to have higher expected returns. Moreover, sorting procedures according to several other price-related ratios yield analogous results. See the survey of Fama and French (2004) for a brief discussion of price-related CAPM-anomalies.

The consumption-based capital asset pricing model (CCAPM) developed by Rubinstein (1976), Lucas (1978) and Breeden (1979) offers one possible explanation for CAPM-anomalies. It suggests that the covariance between an asset's return and the pricing kernel (also called stochastic discount factor) should be used as a measure for an asset's risk. In this framework, the CAPM holds under restrictive assumptions on either the utility function or the distribution of returns. ${ }^{1}$ For example in case of quadratic utility, the pricing kernel becomes a linear function of the market return, and the risk measure of the CCAPM reduces to the CAPM beta. In general, however, the pricing kernel is not linear in the market return and higher-order moments affect the cross-section of stock returns.

The question we address in this paper is whether reasonable assumptions on utility functions and the distribution of returns can resolve anomalies

\footnotetext{
${ }^{1}$ For a list of necessary and sufficient conditions to derive the CAPM see Berk (1997).
} 
of the CAPM in the cross-section of stock returns. This study analyzes an example economy with constant relative risk aversion (CRRA) and lognormal returns. Campbell and Viceira (2002) note that preferences must exhibit CRRA, because there are no long-term trends in risk premia, even though per capita consumption and wealth considerably increased in the past. They also remark that the assumption of lognormal returns allows to capture the non-negativity of gross returns. In case of CRRA preferences and lognormal returns, the pricing kernel is nonlinear and therefore higher-order moments are priced. Our example economy shows that the implied deviations from the CAPM can explain why the security market line is flatter than in the CAPM and why there is a size and a value premium.

Our findings are surprising in light of existing theoretical results in the framework with CRRA preferences and lognormal returns. Merton (1973) finds in a continuous-time model that the CAPM holds when the investment opportunity set is constant through time. Based on this result, Campbell and Viceira (2002) argue that in discrete time an approximate CAPM holds when time intervals are short. Our example economy in a discrete-time framework shows that CAPM-anomalies can become substantial for certain parameter specifications. We find that the coefficient of relative risk aversion and the volatility of fundamentals have to be rather high to get sizeable CAPManomalies, which suggests that the CAPM-puzzles are closely linked to the equity premium puzzle. Thus, the continuous-time framework is technically

more convenient, but it comes at the cost of missing an important feature of the model, namely that it can qualitatively explain CAPM-anomalies in the cross-section of stock returns.

In Section 3, we introduce the model and the underlying assumptions. Section 4 introduces the pricing error of the CAPM in the framework of the CCAPM. Section 5 discusses the flatness of the security market line in our setting. In Section 6, we address the impact of price-related sorting procedures. Section 7 analyzes the comparative statics of the pricing error.

\section{Literature related to the CCAPM with CRRA preferences}

Kraus and Litzenberger (1983) provide conditions on agents' preferences and the distribution of returns that give rise to a three-moment CAPM, which besides mean and variance also incorporates skewness. They show that there is a preference for positive skewness when agents have monotone increasing strictly concave utility functions with nonincreasing absolute risk 
aversion. Higher-order moments are not considered, since preferences for higher-order moments are not completely determined by these assumptions. While assets that follow linear characteristic lines are priced according to the standard CAPM, assets with quadratic characteristic lines are then priced according to a three-moment CAPM. ${ }^{2}$ Harvey and Siddique (2000) test a conditional version of the three-moment CAPM. They find that conditional skewness is significant even in the presence of factors based on size and bookto-market ratio. Furthermore, they note that the size and the value factor partly capture similar information as conditional skewness.

Dittmar (2002) argues in favor of a four-moment CAPM. He shows that positive and decreasing marginal utility, decreasing absolute risk aversion and decreasing absolute prudence imply preference for skewness and kurtosis aversion. He finds that factors like size and value become insignificant when skewness and kurtosis are taken into account. Dittmar (2002) also compares models with a general linear, quadratic and cubic pricing kernel with and without the factors of Fama and French (1993) based on size and book-tomarket ratio to a model with power utility by testing the Euler equation on industry-sorted portfolios. His tests reject the model with CRRA preferences, even though they exhibit positive and decreasing marginal utility, decreasing absolute risk aversion and decreasing absolute prudence.

Dittmar (2002) discusses only preferences for skewness and kurtosis in the theoretical part of his study, since the preferences for higher-order terms are not determined by the mere assumption of positive and decreasing marginal utility, decreasing absolute risk aversion and decreasing absolute prudence. The assumption of power utility also places restrictions on higher-order terms, which Dittmar (2002) does not consider in the theoretical part of his study.

In contrast to Kraus and Litzenberger (1983) and Dittmar (2002), our results implicitly account for all higher-order moments, since our example economy can be solved in closed form. Our study also differs from the study of Dittmar (2002) in that we discuss analytically the impact of assuming CRRA preferences on the size and the value premium, whereas he compares empirically the CCAPM with CRRA preferences to the Fama-French model. Compared to the empirical study on CRRA preferences of Dittmar (2002), our example economy allows us to explore the qualitative properties of the

\footnotetext{
${ }^{2}$ Characteristic lines describe the reaction of asset returns on changes in the market return.
} 
model and gain deeper insights into possible reasons for the quantitative shortcomings of the model. In addition, we analyze the premium on lowbeta assets as well and provide a more integrated view of different CAPManomalies.

\section{The model}

This section introduces a two-period economy with CRRA preferences and lognormal dividends. The two-period framework allows us to discuss the effect of sorting assets into portfolios according to first-period variables. Models with CRRA preferences and lognormal returns are difficult to solve in general. The problem is that the distribution of aggregate dividends, which is the sum of lognormal distributions, is not known. In order to obtain closed-form solutions, we impose a specific correlation structure between the dividends that assets pay.

\subsection{Financial assets}

There are two time-periods $t=0,1$ in the model. The economy consists of a continuum of assets indexed by $\rho \in \mathbb{R}$. The price of asset $\rho$ at time $t=0$ is denoted by $q^{\rho}$. Asset $\rho$ pays a dividend

$$
D_{0}^{\rho}=e^{\rho y_{0}}
$$

at time $t=0$ and

$$
D_{1}^{\rho}=e^{\rho y_{1}}
$$

at time $t=1$, where $y_{0}$ is a constant and

$$
y_{1} \sim \mathcal{N}\left(\mu, \sigma^{2}\right)
$$

The random variable $y_{1}$ models a source of systematic risk. For simplicity we do not model idiosyncratic risk, but we discuss the impact of idiosyncratic risk on the results in Section 6.

The asset-specific parameter $\rho$ is assumed to be normally distributed in the cross-section of assets. More specifically we assume

$$
\rho \sim \mathcal{N}\left(\mu_{\rho}, \sigma_{\rho}^{2}\right)
$$

This assumption generates a quite realistic cross-section of dividends. ${ }^{3}$

\footnotetext{
${ }^{3}$ Elmiger (2010) documents that the cross-section of dividends is approximately lognormally distributed except for the tails of the distribution.
} 
For our derivations we also need the distribution of aggregate dividends. Aggregate dividends at time $t=0$ are given by

$$
D_{0}^{M}=\int_{-\infty}^{\infty} D_{0}^{\rho} f(\rho) d \rho=\int_{-\infty}^{\infty} e^{\rho y_{0}} f(\rho) d \rho=e^{\mu_{\rho} y_{0}+\frac{1}{2} \sigma_{\rho}^{2} y_{0}^{2}}
$$

where $f(\rho)$ denotes the density function of the normal distribution $\mathcal{N}\left(\mu_{\rho}, \sigma_{\rho}^{2}\right)$. Aggregate dividends at time $t=1$ are

$$
D_{1}^{M}=\int_{-\infty}^{\infty} D_{1}^{\rho} f(\rho) d \rho=\int_{-\infty}^{\infty} e^{\rho y_{1}} f(\rho) d \rho=e^{\mu_{\rho} y_{1}+\frac{1}{2} \sigma_{\rho}^{2} y_{1}^{2}}
$$

We see that even though the distribution of the sum of lognormal dividends is not known in general, we can compute aggregate dividends in closed form in this particular case due to our assumption on the correlation structure between dividends. For a very small cross-sectional dispersion of dividends $\sigma_{\rho}^{2}$, which means that the dividend payments of most assets are almost the same, aggregate dividends are approximately lognormally distributed.

\subsection{The representative agent}

There is a representative agent who is initially endowed with all assets in the economy. The agent chooses portfolio holdings $\theta^{\rho}$ to maximize the expected utility derived from consumption $c_{t}$ at time $t=0,1$. The objective function is

$$
u\left(c_{0}\right)+\delta \mathbf{E}\left[u\left(c_{1}\right)\right]
$$

subject to the budget constraints

$$
\begin{aligned}
c_{0}+\int_{-\infty}^{\infty} q^{\rho} \theta^{\rho} d \rho & =\int_{-\infty}^{\infty}\left(q^{\rho}+D_{0}^{\rho}\right) f(\rho) d \rho \\
c_{1} & =\int_{-\infty}^{\infty} D_{1}^{\rho} \theta^{\rho} d \rho .
\end{aligned}
$$

The agent has CRRA preferences $u(c)=(c)^{1-\gamma} /(1-\gamma)$, where $\gamma$ denotes the coefficient of relative risk aversion. In equilibrium the agent has to hold all assets and consumption equals $c_{0}=D_{0}^{M}$ at time $t=0$ and $c_{1}=D_{1}^{M}$ at time $t=1$. 


\section{The pricing error of the CAPM}

The CAPM does not follow from the CCAPM when preferences have CRRA if we do not place certain restrictions on the distribution of returns. This section repeats the difference between the predictions of the CAPM and the CCAPM in case of CRRA preferences ${ }^{4}{ }^{4}$ which we refer to as the pricing error of the CAPM.

In our setting, the Euler equations are given by

$$
\mathbf{E}\left[\delta \frac{u^{\prime}\left(c_{1}\right)}{u^{\prime}\left(c_{0}\right)} R^{\rho}\right]=1 \quad \text { for } \rho \in \mathbb{R},
$$

where $u^{\prime}\left(c_{1}\right) / u^{\prime}\left(c_{0}\right)=\left(c_{0} / c_{1}\right)^{\gamma}$. This is equivalent to

$$
\underbrace{\mathbf{E}\left[\delta\left(\frac{c_{0}}{c_{1}}\right)^{\gamma}\right]}_{\frac{1}{R^{f}}} \mathbf{E}\left[R^{\rho}\right]+\operatorname{Cov}\left(\delta\left(\frac{c_{0}}{c_{1}}\right)^{\gamma}, R^{\rho}\right)=1,
$$

or, rearranged,

$$
\mathbf{E}\left[R^{\rho}\right]-R^{f}=-R^{f} \operatorname{Cov}\left(\delta\left(\frac{c_{0}}{c_{1}}\right)^{\gamma}, R^{\rho}\right) .
$$

$R^{f}$ denotes the return on the riskless asset that pays 1 at $t=1$ with certainty. Since the above equation also holds for the market portfolio, we can write

$$
\frac{\mathbf{E}\left[R^{\rho}\right]-R^{f}}{\mathbf{E}\left[R^{M}\right]-R^{f}}=\frac{\operatorname{Cov}\left(c_{1}^{-\gamma}, R^{\rho}\right)}{\operatorname{Cov}\left(c_{1}^{-\gamma}, R^{M}\right)}=\frac{\operatorname{Cov}\left(\left(R^{M}\right)^{-\gamma}, R^{\rho}\right)}{\operatorname{Cov}\left(\left(R^{M}\right)^{-\gamma}, R^{M}\right)},
$$

where $R^{M}$ denotes the return on the market portfolio. The second equation follows, because the consumption of the representative agent equals aggregate dividends in equilibrium. Multiplying the numerator and denominator with the price of the market portfolio $q^{M}$ to the power of $\gamma$ leads to the above expression.

We can write the above equation equivalently as

$$
\mathbf{E}\left[R^{\rho}\right]-R^{f}=\alpha^{\rho}+\beta^{\rho}\left(\mathbf{E}\left[R^{M}\right]-R^{f}\right),
$$

\footnotetext{
${ }^{4}$ The derivations can be found for example in LeRoy and Werner (2001).
} 
where

$$
\beta^{\rho}=\frac{\operatorname{Cov}\left(R^{\rho}, R^{M}\right)}{\operatorname{Var}\left(R^{M}\right)}
$$

and

$$
\alpha^{\rho}=\underbrace{\left(\frac{\operatorname{Cov}\left(\left(R^{M}\right)^{-\gamma}, R^{\rho}\right)}{\operatorname{Cov}\left(\left(R^{M}\right)^{-\gamma}, R^{M}\right)}-\frac{\operatorname{Cov}\left(R^{\rho}, R^{M}\right)}{\operatorname{Var}\left(R^{M}\right)}\right)}_{\equiv A^{\rho}}\left(\mathbf{E}\left[R^{M}\right]-R^{f}\right) .
$$

If $\alpha^{\rho}$ were zero for all $\rho \in \mathbb{R}$, we would obtain the CAPM. The term $\alpha^{\rho}$ therefore denotes the pricing error of the CAPM with respect to asset $\rho$ in our model.

From the expression above we see that the size of the pricing error depends on the expected market excess return, which is known to be too low in these kinds of models. However, the market clearing condition sets consumption equal to aggregate dividends. For illustration purposes, we use parameter values in accordance with dividend data in the following. Dividend data is more volatile than consumption data. Therefore we need lower coefficients of relative risk aversion to generate high equity premia. We then discuss in Section 7 how sensitive the results are to consumption growth parameters.

\section{The smaller slope of the security market line}

Empirical studies like Black et al. (1972) find that the security market line estimated from the data is flatter than the CAPM predicts. Low-beta stocks offer a premium that is too high and high-beta stocks offer a premium that is too low. The following analysis shows that the pricing error of the CAPM exhibits the same characteristics in our model.

\subsection{The case of a small cross-sectional dispersion}

This section examines a simplified version of the model. We assume that the distribution of aggregate dividends at time $t=1$ approximately follows a lognormal distribution

$$
D_{1}^{M} \approx e^{y_{1}}
$$

This assumption is reasonable for very small dispersions of cross-sectional dividends $\sigma_{\rho}^{2}$. The model then becomes more tractable and allows for a 
better understanding of the properties of the pricing error. The next section addresses the generalization of our results to economies with arbitrarily large dispersions of dividends. The parameter $\mu_{\rho}$ has no impact on the qualitative properties of the pricing error, since it only shifts the normal distribution. Therefore, aggregate dividends would still follow a lognormal distribution for $\mu_{\rho}$ different from one as long as the cross-sectional dispersion is zero.

The pricing error in the simplified model is given by

$$
\alpha^{\rho}=A^{\rho}\left(\mathbf{E}\left[R^{M}\right]-R^{f}\right),
$$

where

$$
A^{\rho}=\frac{e^{-\gamma \sigma^{2}}}{e^{-\rho \gamma \sigma^{2}}}\left(\frac{e^{-\rho \gamma \sigma^{2}}-1}{e^{-\gamma \sigma^{2}}-1}-\frac{e^{\rho \sigma^{2}}-1}{e^{\sigma^{2}}-1}\right)
$$

and

$$
\mathbf{E}\left[R^{M}\right]-R^{f}=\frac{1}{\delta} e^{\gamma\left(\mu-\ln \left(c_{0}\right)\right)-\frac{1}{2} \gamma^{2} \sigma^{2}}\left(e^{\gamma \sigma^{2}}-1\right) .
$$

Appendix B and Appendix $\mathrm{C}$ derive the pricing error in the model without the lognormal approximation of aggregate dividends. Setting $\mu_{\rho}=1$ and $\sigma_{\rho}=0$, we obtain the expression of the pricing error given above. We observe that the expected market excess return only scales the pricing error. Cross-sectional differences in mispricing are entirely driven by $A^{\rho}$. The above expression also shows that the pricing error is zero for values of $\rho \in\{0,1\}$, since the CAPM holds for the riskless asset and the market portfolio.

Appendix D shows that the function $A^{\rho}$ and therefore the pricing error $\alpha^{\rho}$ decreases in $\rho$ for values of $\rho$ larger than

$$
\rho_{A}^{\max }=\frac{1}{\sigma^{2}} \ln \left(\frac{\gamma\left(e^{-\gamma \sigma^{2}}-e^{\sigma^{2}}\right)}{(\gamma+1)\left(e^{-\gamma \sigma^{2}}-1\right)}\right),
$$

and increases in $\rho$ for smaller values of $\rho$.

Next, we assess the CAPM beta $\beta^{\rho}$ as a function of $\rho$. The CAPM beta in our simplified model is given by

$$
\beta^{\rho}=\frac{e^{-\gamma \sigma^{2}}\left(e^{\rho \sigma^{2}}-1\right)}{e^{-\rho \gamma \sigma^{2}}\left(e^{\sigma^{2}}-1\right)} .
$$

Appendix $\mathrm{B}$ shows the derivation of $\beta^{\rho}$ in the general model without the lognormal approximation of aggregate dividends. Setting $\mu_{\rho}=1$ and $\sigma_{\rho}=0$, 

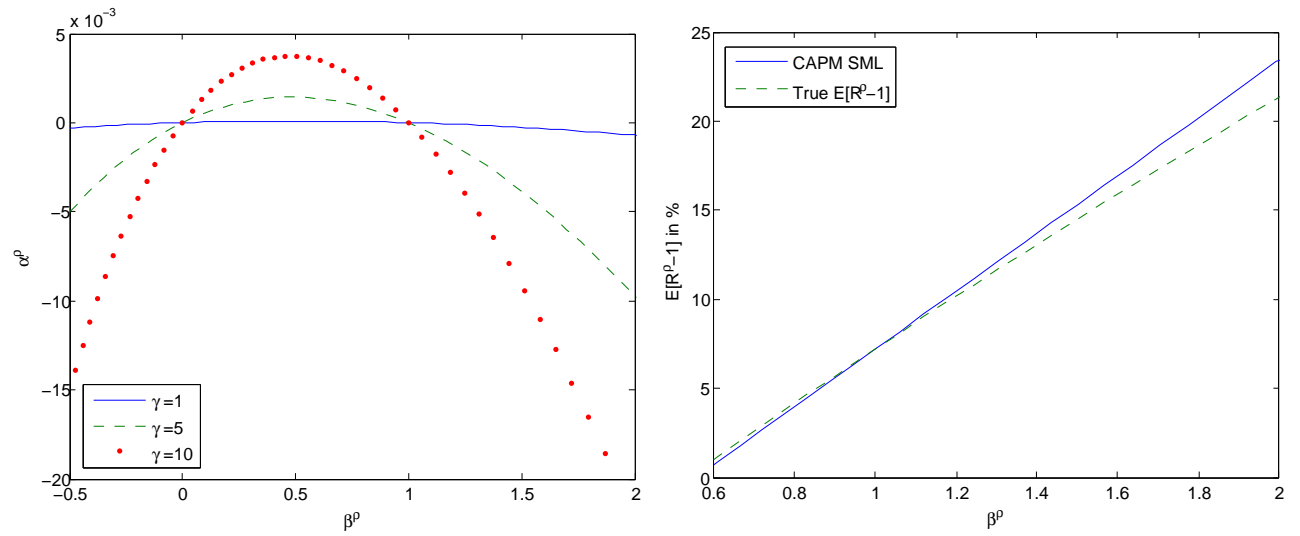

Figure 1: The left plot shows the pricing error as a function of the CAPM beta for different coefficients of relative risk aversion. The right plot shows the security market line of the CAPM and true expected stock returns as a function of the CAPM beta for a coefficient of relative risk aversion of 9. The discount factor $\delta$ is set to 0.96 , mean logarithmic aggregate dividend growth $\mu-\ln \left(c_{0}\right)$ is set to $6.7 \%$ and the standard deviation of logarithmic dividend growth $\sigma$ is set to $13.5 \%$.

we obtain the expression above. The beta of the riskless asset $\rho=0$ is zero and the beta of the market portfolio $\rho=1$ is one as expected. Appendix E shows that the CAPM beta is increasing in $\rho$ for values of $\rho$ larger than

$$
\rho_{\beta}^{\min }=\frac{1}{\sigma^{2}} \ln \left(\frac{\gamma}{\gamma+1}\right)
$$

and decreasing in $\rho$ for smaller values of $\rho$. Appendix $\mathrm{F}$ graphically shows the factor $A^{\rho}$ of the pricing error and the CAPM beta as a function of $\rho$.

The above analytical results suggest that the pricing error is a decreasing function of the CAPM beta for values of $\beta^{\rho}$ larger than $\beta_{\beta}^{\rho^{\min }}$. The left plot in Figure 1 illustrates the relation between the pricing error and the CAPM beta for different coefficients of relative risk aversion. We see that low-beta assets have indeed a positive pricing error in our model, whereas high-beta assets have a negative pricing error for values of beta around 0.5 and above. Since we assume a very small cross-sectional dispersion of dividends, the fraction of assets in the increasing part of the curve $\alpha^{\rho}$, where this relation breaks down, is negligibly small. The plots in Figure 1 assume a discount factor of 0.96 , mean logarithmic dividend growth of $6.7 \%$ and a standard 
deviation of logarithmic dividend growth of $13.5 \% .^{5}$

The right plot of Figure 1 compares the expected net returns of assets to the security market line predicted by the CAPM. We see that the expected returns of assets with a CAPM beta that is larger than 0.6 lie on an almost straight line that is flatter than the security market line predicted by the CAPM. The left plot shows that the slope of the security market line becomes even smaller for larger coefficients of relative risk aversion. Section 7 provides more details on the comparative statics.

\subsection{The more general model}

The simplified model of the previous section suggests that the security market line is flatter in the CCAPM with CRRA preferences than in the CAPM over a specific range of assets. In case of a very small cross-sectional dispersion of dividends, pretty much all assets lie in this range. This section checks if the previous results carry over to the more general model that allows for any cross-sectional dispersion of dividends.

Appendix B and Appendix C derive the pricing error as well as the CAPM beta of the more general model in closed form. Now let us compare economies that differ in their cross-sectional distribution of dividends. Recall that the cross-section of dividends is given by

$$
D_{1}^{\rho}=e^{\rho y_{1}}
$$

where

$$
\rho \sim \mathcal{N}\left(\mu_{\rho}, \sigma_{\rho}^{2}\right)
$$

The cross-section is therefore completely characterized by the parameters $\mu_{\rho}$ and $\sigma_{\rho}^{2}$. Note that both parameters affect the distribution of aggregate dividends, which is given by

$$
D_{1}^{M}=e^{\mu_{\rho} y_{1}+\frac{1}{2} \sigma_{\rho}^{2} y_{1}^{2}} .
$$

In order to obtain comparable plots, we match the first two moments of logarithmic consumption growth for each pair of values $\mu_{\rho}$ and $\sigma_{\rho}$. In the previous section we assumed $\mu-\ln \left(c_{o}\right)=6.7 \%$ and $\sigma=13.5 \%$ for the parameters $\mu_{\rho}=1$ and $\sigma_{\rho}=0$. In addition, we assume that initial consumption equals $c_{0}=10000$ units. For the derivation of the first and second moment of logarithmic consumption growth see Appendix H.

\footnotetext{
${ }^{5}$ The chosen parameters for the distribution of aggregate dividend growth are supported by the empirical study of Chen (2009).
} 

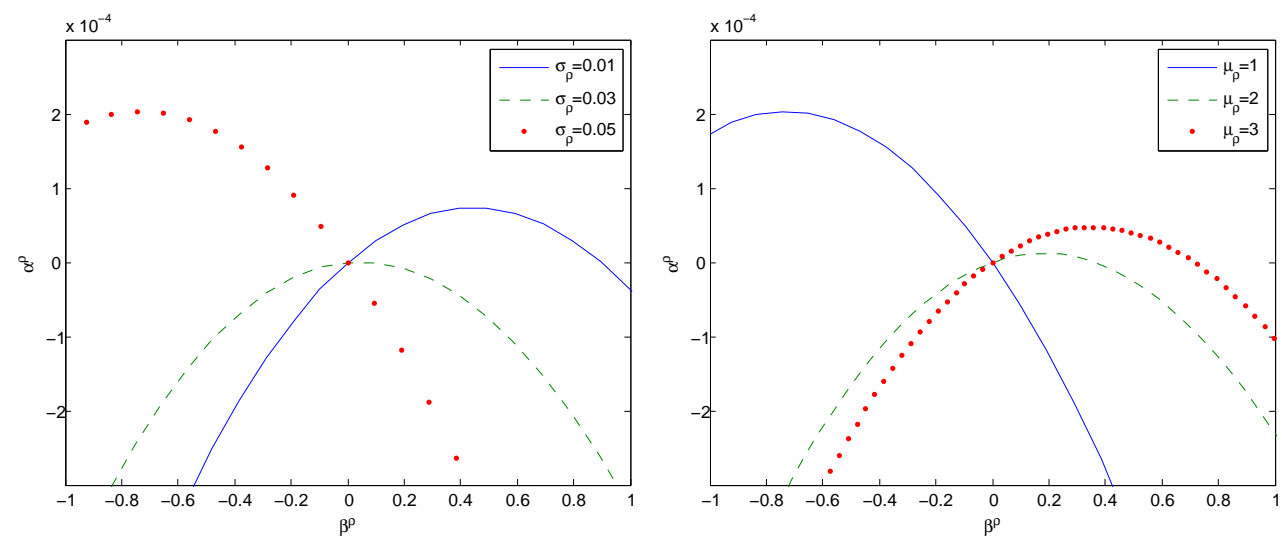

Figure 2: The two plots show the pricing error $\alpha^{\rho}$ as a function of the CAPM beta $\beta^{\rho}$ for different values of $\mu_{\rho}$ and $\sigma_{\rho}^{2}$. The left plot assumes $\mu_{\rho}=1$ and the right plot assumes $\sigma_{\rho}=0.05$.

\begin{tabular}{|l|r|r|r|r|r|}
\hline$\mu_{\rho}$ & 1 & 2 & 3 & 1 & 1 \\
$\sigma_{\rho}$ & 0.05 & 0.05 & 0.05 & 0.01 & 0.03 \\
\hline$\beta^{\rho}$ percentile & 0.8606 & 0.9348 & 0.9577 & 0.9752 & 0.9206 \\
\hline
\end{tabular}

Table 1: First percentile of beta for different parameter values $\mu_{\rho}$ and $\sigma_{\rho}$.

Figure 2 compares the pricing error $\alpha^{\rho}$ for different values of $\mu_{\rho}$ and $\sigma_{\rho}^{2}$ assuming log utility. We observe that the shape of the curve remains basically the same in the sense that the curve reaches a maximum and then starts to decrease for increasing values of $\beta^{\rho}$. The left plot assumes $\mu_{\rho}=1$ and the right plot assumes $\sigma_{\rho}=0.05$. Note that the pricing error becomes maximal for a $\beta$ smaller than one. Most assets lie in the decreasing part of the curve, which suggests that the security market line is too flat over a large range of assets. To illustrate this point, Table 1 shows the first percentile of beta values for different values of $\mu_{\rho}$ and $\sigma_{\rho}^{2}$. We observe that the first percentile lies clearly in the decreasing part of the pricing error curve. Except for a very small number of assets, there is a decreasing relation between the pricing error and the CAPM beta. The security market line is therefore too flat over a large range of assets. 


\section{Price-related sorts and the role of idiosyncratic risk}

Empirical evidence suggests that deviations from the traditional CAPM become particularly clear when stocks are sorted into portfolios according to market capitalization or book-to-market ratios. Before we move to pricerelated sorting procedures, we discuss the impact of idiosyncratic risk on the results. We show that our previous results are unaffected by the introduction of idiosyncratic risk, but idiosyncratic risk does affect prices and thus pricerelated sorts.

\subsection{Idiosyncratic risk}

In a first step, let us introduce idiosyncratic risk into the model. We assume that dividends are given by

$$
D_{1}^{\rho}=e^{\rho y_{1}+\epsilon_{\rho}},
$$

where $\epsilon_{\rho}$ denotes an asset-specific source of risk. Prices then are

$$
q^{\rho}=\mathbf{E}\left[\delta\left(\frac{c_{0}}{c_{1}}\right)^{\gamma} D_{1}^{\rho}\right]=\mathbf{E}\left[\delta\left(\frac{c_{0}}{c_{1}}\right)^{\gamma} e^{\rho y_{1}+\epsilon_{\rho}}\right]=\mathbf{E}\left[\delta\left(\frac{c_{0}}{c_{1}}\right)^{\gamma} e^{\rho y_{1}}\right] \mathbf{E}\left[e^{\epsilon_{\rho}}\right] .
$$

We see that prices depend on the expectation of the idiosyncratic risk factor. Idiosyncratic risk therefore matters when we discuss price-related sorting procedures. On the other hand, idiosyncratic risk has no impact on the CAPM beta and the pricing error, since they only depend on systematic risk. Let us illustrate this point briefly in mathematical terms. The CAPM beta in a model with idiosyncratic risk is given by

$$
\beta^{\rho}=\frac{\operatorname{Cov}\left(R^{\rho}, R^{M}\right)}{\operatorname{Var}\left(R^{M}\right)}=\frac{\operatorname{Cov}\left(\frac{e^{\rho y_{1}+\epsilon_{\rho}}}{q^{\rho}}, R^{M}\right)}{\operatorname{Var}\left(R^{M}\right)}=\frac{\operatorname{Cov}\left(e^{\rho y_{1}}, R^{M}\right) \frac{\mathbf{E}\left[e^{\epsilon \rho}\right]}{q^{\rho}}}{\operatorname{Var}\left(R^{M}\right)} .
$$

Inserting the above expression for prices, we obtain

$$
\beta^{\rho}=\frac{\operatorname{Cov}\left(e^{\rho y_{1}}, R^{M}\right) \mathbf{E}\left[\delta\left(\frac{c_{0}}{c_{1}}\right)^{\gamma} e^{\rho y_{1}}\right]^{-1}}{\operatorname{Var}\left(R^{M}\right)} .
$$

We observe that $\beta^{\rho}$ is unaffected by idiosyncratic risk. For computing $\alpha^{\rho}$ we proceed analogously. Recall that the pricing error is given by

$$
\alpha^{\rho}=\left(\frac{\operatorname{Cov}\left(\left(R^{M}\right)^{-\gamma}, R^{\rho}\right)}{\operatorname{Cov}\left(\left(R^{M}\right)^{-\gamma}, R^{M}\right)}-\frac{\operatorname{Cov}\left(R^{\rho}, R^{M}\right)}{\operatorname{Var}\left(R^{M}\right)}\right)\left(\mathbf{E}\left[R^{M}\right]-R^{f}\right) .
$$


The only two terms that could possibly depend on idiosyncratic risk are $\operatorname{Cov}\left(\left(R^{M}\right)^{-\gamma}, R^{\rho}\right)$ and $\operatorname{Cov}\left(R^{\rho}, R^{M}\right)$. The second term does not depend on idiosyncratic risk as shown above and

$$
\operatorname{Cov}\left(\left(R^{M}\right)^{-\gamma}, R^{\rho}\right)=\operatorname{Cov}\left(\left(R^{M}\right)^{-\gamma}, \frac{e^{\rho y_{1}+\epsilon_{\rho}}}{q^{\rho}}\right)=\operatorname{Cov}\left(\left(R^{M}\right)^{-\gamma}, e^{\rho y_{1}}\right) \frac{\mathbf{E}\left[e^{\epsilon_{\rho}}\right]}{q^{\rho}}
$$

Inserting the above expression for prices, we have

$$
\operatorname{Cov}\left(\left(R^{M}\right)^{-\gamma}, R^{\rho}\right)=\operatorname{Cov}\left(\left(R^{M}\right)^{-\gamma}, e^{\rho y_{1}}\right) \mathbf{E}\left[\delta\left(\frac{c_{0}}{c_{1}}\right)^{\gamma} e^{\rho y_{1}}\right]^{-1} .
$$

The pricing error $\alpha^{\rho}$ as well as the CAPM beta are therefore unaffected by idiosyncratic risk. Since the CAPM beta as well as the pricing error do not depend on idiosyncratic risk, we conclude that our previous results in a model without idiosyncratic risk still hold in the presence of idiosyncratic risk. However, idiosyncratic risk affects prices and therefore plays a role in the following discussion of price-related sorting procedures.

\subsection{Price-related sorting procedures}

The preceding discussion on idiosyncratic risk shows that a sort according to market capitalization cannot perfectly sort according to abnormal returns in general, because idiosyncratic risk affects prices but not abnormal returns. Results on price-related sorting procedures therefore depend on idiosyncratic risk. In our analysis, we do not model idiosyncratic risk explicitly. Instead we stick to our model without idiosyncratic risk for the derivation of the results, based on which we then discuss the impact of idiosyncratic risk. In addition, we restrict ourselves to the more tractable version of the model with a small cross-sectional dispersion of dividends, where aggregate dividends are approximately lognormally distributed.

First, let us discuss sorting according to market capitalization $q^{\rho}$ and the size premium. ${ }^{6}$ Appendix $G$ shows that the market capitalization increases in $\rho$ for values of $\rho$ larger than

$$
\rho_{q}^{\min }=\gamma-\frac{\mu}{\sigma^{2}}
$$

Since the pricing error is a decreasing function in $\rho$ for values of $\rho$ larger than $\rho_{A}^{\max }$, the pricing error also decreases in size for values of $\rho$ that are larger than $\rho_{q}^{\min }$ and $\rho_{A}^{\max }$.

\footnotetext{
${ }^{6}$ Note that our model makes no distinction between market capitalization and prices.
} 

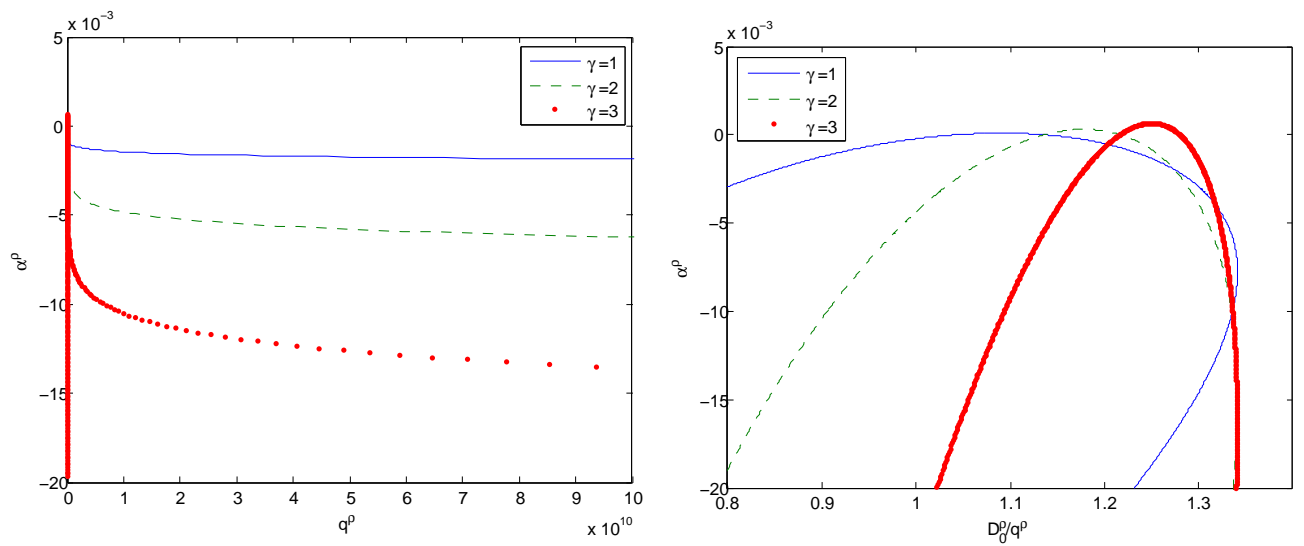

Figure 3: The left plot shows the relation between market capitalization and the pricing error for different coefficients of relative risk aversion. The right plot shows the relation between the dividend-price ratio and the pricing error. The discount factor $\delta$ is set to 0.96 , mean logarithmic aggregate dividend growth $\mu-\ln \left(c_{0}\right)$ is set to $6.7 \%$, the standard deviation of logarithmic dividend growth $\sigma$ is set to $13.5 \%$ and initial consumption $c_{0}$ is set to 10000 units.

The left plot of Figure 3 illustrates graphically how the pricing error relates to market capitalization according to our model. In contrast to the CAPM beta and the pricing error, market capitalization does depend on consumption in the first period. The plots in Figure 3 assume $c_{0}=10000$ units. Since we normalized the total number of assets to one, $c_{0}$ denotes average dividends. We consider three different levels of relative risk aversion. For $\gamma=1(2,3)$, we find that $\rho_{q}^{\min }=-509.6360(-508.6360,-507.6360)$ and $\rho_{A}^{\max }=0.5023(0.5038,0.5053)$. Note that the number of assets that lie on the increasing left part of the pricing error curve $\alpha^{\rho}$ is very small if the crosssectional dispersion of dividends is small. We can therefore say that small stocks tend to pay a higher premium than the CAPM implies and large stocks pay a lower premium than in the CAPM.

The right plot of Figure 3 compares value stocks to growth stocks as measured by the dividend-price ratio. For a small cross-sectional dispersion of dividends, there are only few stocks on the very right of the curve. The plot therefore suggests that stocks with a high dividend-price ratio tend to pay a higher premium than the CAPM implies and stocks with a low dividend-price ratio pay a lower premium than in the CAPM. 
The previous analyses allow no general conclusions for the model with idiosyncratic risk. In the presence of idiosyncratic risk, the relation between prices or price-related ratios and the pricing error is not clear if we do not put further assumptions. Depending on the distribution of idiosyncratic risk across assets, the results could look very different. However, if we assume that the expectation of the idiosyncratic risk factor is equal for all assets, then all prices are scaled by the same factor and the results remain qualitatively the same. Accordingly, if the differences in the expectation of the idiosyncratic risk factor do not differ too much across assets, we would at least expect the previous results to hold for sorted portfolios of assets.

\section{Comparative Statics}

The previous results show that the security market line is flatter in our model than in the CAPM, which leads to price-related CAPM-anomalies in the cross-section of returns. This section analyzes how the CAPM pricing errors depend on market conditions. Recall that the pricing error in the simplified model with lognormal aggregate dividends is given by

$$
\alpha^{\rho}=A^{\rho}\left(\mathbf{E}\left[R^{M}\right]-R^{f}\right),
$$

where

$$
A^{\rho}=\frac{e^{-\gamma \sigma^{2}}}{e^{-\rho \gamma \sigma^{2}}}\left(\frac{e^{-\rho \gamma \sigma^{2}}-1}{e^{-\gamma \sigma^{2}}-1}-\frac{e^{\rho \sigma^{2}}-1}{e^{\sigma^{2}}-1}\right)
$$

and

$$
\mathbf{E}\left[R^{M}\right]-R^{f}=\frac{1}{\delta} e^{\gamma\left(\mu-\ln \left(c_{0}\right)\right)-\frac{1}{2} \gamma^{2} \sigma^{2}}\left(e^{\gamma \sigma^{2}}-1\right)
$$

From the above expression we see that the pricing error depends on four parameters: the aggregate dividend growth parameters $\mu-\ln \left(c_{0}\right)$ and $\sigma^{2}$ and the preference parameters $\gamma$ and $\delta$.

\subsection{Aggregate dividend growth parameters}

The impact of mean logarithmic aggregate dividend growth $\mu-\ln \left(c_{0}\right)$ on the pricing error is immediately clear from the above expression for the pricing error (1). It only affects the pricing error through the equity premium. The higher $\mu-\ln \left(c_{0}\right)$ is, the larger the market premium and therefore the pricing error is. In times of high expected logarithmic dividend growth we 

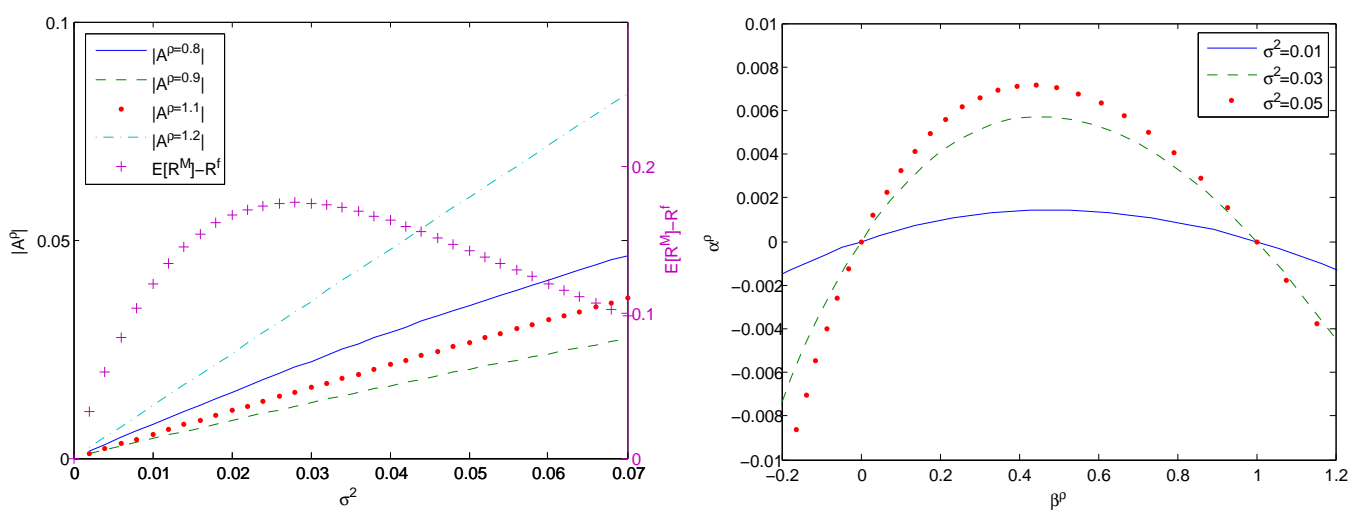

Figure 4: The left plot shows the absolute value of the pricing error factor $A^{\rho}$ and the expected market excess return as a function of the variance of logarithmic aggregate dividend growth for different assets. The right plot shows the pricing error as a function of the CAPM beta for different variances. The discount factor $\delta$ is set to 0.96 , mean logarithmic aggregate dividend growth $\mu-\ln \left(c_{0}\right)$ is set to $6.7 \%$ and the coefficient of relative risk aversion is set to 9 .

therefore expect cross-sectional anomalies to be more pronounced than in times of low expected logarithmic dividend growth.

The impact from the variance of logarithmic aggregate dividend growth $\sigma^{2}$ is more complex. Note that the pricing error consists of two components: the factor $A^{\rho}$ and the expected market excess return. Figure 4 plots them separately and shows the resulting pricing error. From the left plot we see that the absolute value of $A^{\rho}$ increases with $\sigma^{2}$. The expected market excess return also increases over a certain range of values, but starts to decrease for larger values of $\sigma^{2}$. Overall, we can say that asset anomalies become more pronounced for larger values of $\sigma^{2}$ as long as $\sigma^{2}$ is not too large. The right plot of Figure 4 shows the relation between the pricing error and the CAPM beta for different variances of logarithmic dividend growth $\sigma^{2}$ and illustrates this point.

\subsection{Preference parameters}

The effect of the time discount factor on the pricing error can be directly seen from the above expression for the pricing error (1). The time discount factor has no impact on $A^{\rho}$ and only affects the equity premium. The more impatient the representative agent is, the higher the market premium and 
therefore the pricing error is. This finding is supported by empirical evidence. The empirical study of Caliskan and Hens (2013) shows for example that the value premium is more pronounced in countries with more impatient investors.
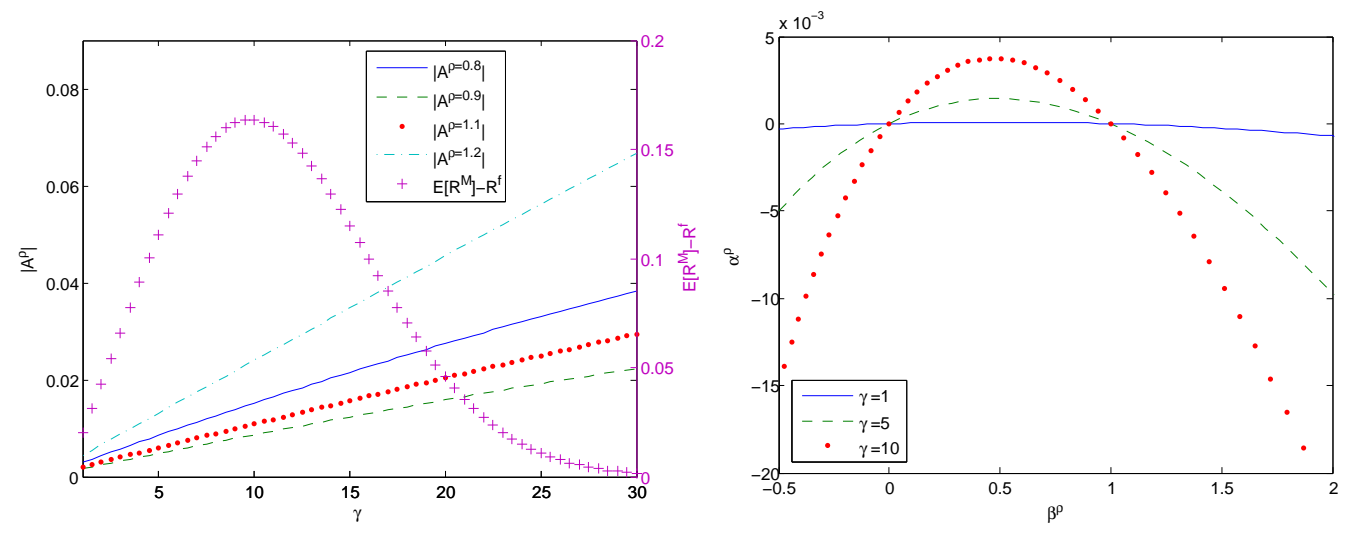

Figure 5: The left plot shows the absolute value of the pricing error factor $A^{\rho}$ and the expected market excess return as a function of risk aversion for different assets. The right plot shows the pricing error as a function of the CAPM beta for different coefficients of relative risk aversion. The discount factor $\delta$ is set to 0.96, mean logarithmic aggregate dividend growth $\mu-\ln \left(c_{0}\right)$ is set to $6.7 \%$ and the standard deviation of logarithmic dividend growth $\sigma$ is set to $13.5 \%$.

The effect from the coefficient of relative risk aversion is more complex. The left plot of Figure 5 shows the absolute value of $A^{\rho}$ and the expected market excess return as a function of the risk aversion coefficient. While the pricing error factor $A^{\rho}$ increases for increasing coefficients of relative risk aversion, the expected market excess return is not monotonic. The expected market excess return increases in $\gamma$ for small values of $\gamma$, but decreases in $\gamma$ for larger values of $\gamma$. We conclude that the asset anomalies becomes more pronounced for larger values of relative risk aversion as long as the coefficient of relative risk aversion lies in a reasonable range. The right plot of Figure 5 shows the pricing error as a function of the CAPM beta for different coefficients of relative risk aversion. The empirical study of Caliskan and Hens (2013) also finds support for an increasing relation between the value premium and the coefficient of relative risk aversion. 


\section{Conclusion}

The presented example economy shows that the CCAPM with CRRA preferences and lognormal returns can qualitatively explain CAPM-anomalies in the cross-section of stock returns. The model implies a security market line that is flatter than the one in the CAPM over the range of most assets. Most low-beta assets therefore pay higher abnormal returns than high-beta assets. Furthermore, the smaller slope of the security market line leads to a size premium and a value premium.

The slope of the security market line and thus the size of the CAPManomalies strongly depend on the dividend and preference parameters. The crucial parameters determining cross-sectional differences are the coefficient of relative risk aversion and volatility of aggregate dividend growth. We find that the security market line becomes flatter for increasing risk aversion and increasing volatility. However, the coefficient of relative risk aversion needs to be rather large to get large deviations from the CAPM. In this sense the cross-sectional puzzles are very similar to the equity premium puzzle. The CAPM-puzzles are also tightly linked to the equity premium puzzle, because the pricing errors are proportional to the equity premium. We conclude that in light of our model, the existence of a beta premium, a value premium and a size premium is not a puzzle. It is the magnitude of these premia that is puzzling, and the results argue in favor of a common explanation for the size of the equity premium and the size of CAPM-anomalies.

Our results offer no conclusive answer to the question whether CRRA preferences can explain CAPM-anomalies. There exist different explanations for the size of the equity premium in the equity premium puzzle literature that potentially explain the size of cross-sectional anomalies as well. They are based on different assumptions on preferences or returns. One possible explanation that is consistent with CRRA preferences are rare disasters in the sense of Rietz (1988) and Barro (2006). Including rare disasters to the model has a considerable impact on higher-order moments and decreases for example skewness. Since CRRA preferences imply a preference for positive skewness, a lower skewness in returns leads to a higher equity premium. Rare disasters could explain why the empirical study of Dittmar (2002) finds no support for a CRRA explanation of CAPM-anomalies, since the data does not reflect the risk for rare disasters.

This study also analyzes and compares the beta sort to price-related sorts. While beta only captures systematic risk, prices depend on systematic and 
idiosyncratic risk. However, the pricing error of the CAPM only depends on systematic risk. The relation between the pricing error and price-related sorts is therefore disturbed by idiosyncratic risk.

Our discussion of CAPM-anomalies in the cross-section of stock returns is based on a simple example economy. An interesting extension would be to derive more general conditions on the distribution of returns under which CAPM-anomalies arise. This could serve as a basis for a more integrated view of the equity premium puzzle and the CAPM-puzzles. 


\section{Appendix A. Derivation of the expectation}

In the following we often have to compute expectations of the form

$$
\mathbf{E}\left[e^{a y_{1}+b y_{1}^{2}}\right]
$$

for coefficients $a$ and $b$. Using the completion of the square method we obtain

$$
\begin{aligned}
\mathbf{E}\left[e^{a y_{1}+b y_{1}^{2}}\right] & =\frac{1}{\sigma \sqrt{2 \pi}} \int_{-\infty}^{\infty} e^{a y+b y^{2}} e^{-\frac{(y-\mu)^{2}}{2 \sigma^{2}}} d y \\
& =\frac{1}{\sigma \sqrt{2 \pi}} \int_{-\infty}^{\infty} e^{-\frac{1}{2}\left(\frac{1}{\sigma^{2}}-2 b\right)\left(y^{2}-2 \frac{a \sigma^{2}+\mu}{1-2 b \sigma^{2}} y+\frac{\mu^{2}}{1-2 b \sigma^{2}}\right)} d y \\
& =\frac{1}{\sigma \sqrt{2 \pi}} e^{-\frac{1}{2}\left(\frac{1}{\sigma^{2}}-2 b\right)\left(\frac{\mu^{2}}{1-2 b \sigma^{2}}-\left(\frac{a \sigma^{2}+\mu}{1-2 b \sigma^{2}}\right)^{2}\right)} \int_{-\infty}^{\infty} e^{-\frac{1}{2}\left(\frac{1}{\sigma^{2}}-2 b\right)\left(y-\frac{a \sigma^{2}+\mu}{1-2 b \sigma^{2}}\right)^{2}} d y
\end{aligned}
$$

The integrand is proportional to a normal density function with mean $\frac{a \sigma^{2}+\mu}{1-2 b \sigma^{2}}$ and standard deviation $\sqrt{\frac{\sigma^{2}}{1-2 b \sigma^{2}}}$. The expectation therefore is equal to

$$
\begin{aligned}
\mathbf{E}\left[e^{a y_{1}+b y_{1}^{2}}\right] & =\frac{1}{\sqrt{1-2 b \sigma^{2}}} e^{-\frac{1}{2}\left(\frac{1}{\sigma^{2}}-2 b\right)\left(\frac{\mu^{2}}{1-2 b \sigma^{2}}-\left(\frac{a \sigma^{2}+\mu}{1-2 b \sigma^{2}}\right)^{2}\right)} \\
& =\frac{1}{\sqrt{1-2 b \sigma^{2}}} e^{-\frac{1}{2} \frac{a^{2} \sigma^{2}+2(a+b \mu) \mu}{2 b \sigma^{2}-1}}
\end{aligned}
$$

\section{Appendix B. Derivation of $A^{\rho}$ and $\beta^{\rho}$}

This section computes

$$
A^{\rho}=\left(\frac{\operatorname{Cov}\left(\left(R^{M}\right)^{-\gamma}, R^{\rho}\right)}{\operatorname{Cov}\left(\left(R^{M}\right)^{-\gamma}, R^{M}\right)}-\frac{\operatorname{Cov}\left(R^{\rho}, R^{M}\right)}{\operatorname{Var}\left(R^{M}\right)}\right),
$$

which can equivalently be written as

$$
A^{\rho}=\frac{q^{M}}{q^{\rho}}\left(\frac{\operatorname{Cov}\left(\left(D_{1}^{M}\right)^{-\gamma}, D_{1}^{\rho}\right)}{\operatorname{Cov}\left(\left(D_{1}^{M}\right)^{-\gamma}, D_{1}^{M}\right)}-\frac{\operatorname{Cov}\left(D_{1}^{\rho}, D_{1}^{M}\right)}{\operatorname{Var}\left(D_{1}^{M}\right)}\right) .
$$


Now let us compute all the covariances in $A^{\rho}$. The numerator of the first summand is

$$
\begin{aligned}
\operatorname{Cov}\left(\left(D_{1}^{M}\right)^{-\gamma}, D_{1}^{\rho}\right) & =\operatorname{Cov}\left(e^{-\gamma\left(\mu_{\rho} y_{1}+\frac{1}{2} \sigma_{\rho}^{2} y_{1}^{2}\right)}, e^{\rho y_{1}}\right) \\
& =\mathbf{E}\left[e^{\left(\rho-\gamma \mu_{\rho}\right) y_{1}-\gamma \frac{1}{2} \sigma_{\rho}^{2} y_{1}^{2}}\right]-\mathbf{E}\left[e^{-\gamma\left(\mu_{\rho} y_{1}+\frac{1}{2} \sigma_{\rho}^{2} y_{1}^{2}\right)}\right] \mathbf{E}\left[e^{\rho y_{1}}\right] .
\end{aligned}
$$

Using the formula in Appendix A for expectations of the above form, we obtain

$$
\operatorname{Cov}\left(\left(D_{1}^{M}\right)^{-\gamma}, D_{1}^{\rho}\right)=C_{1}\left(e^{\frac{1}{2} \frac{\left(\rho^{2}-2 \rho \gamma \mu \rho\right) \sigma^{2}+2 \rho \mu}{\gamma \sigma_{\rho}^{2} \sigma^{2}+1}}-e^{\frac{1}{2}\left(\rho^{2} \sigma^{2}+2 \rho \mu\right)}\right),
$$

where

$$
C_{1}=\frac{1}{\sqrt{1+\gamma \sigma_{\rho}^{2} \sigma^{2}}} e^{\frac{1}{2} \frac{\gamma^{2} \mu_{\rho}^{2} \sigma^{2}-2 \gamma \mu \rho \mu-\gamma \sigma_{\rho}^{2} \mu^{2}}{\gamma \sigma_{\rho}^{2} \sigma^{2}+1}} .
$$

Proceeding accordingly, the covariance in the denominator is

$$
\begin{aligned}
\operatorname{Cov}\left(\left(D_{1}^{M}\right)^{-\gamma}, D_{1}^{M}\right) & =\operatorname{Cov}\left(e^{-\gamma\left(\mu_{\rho} y_{1}+\frac{1}{2} \sigma_{\rho}^{2} y_{1}^{2}\right)}, e^{\mu_{\rho} y_{1}+\frac{1}{2} \sigma_{\rho}^{2} y_{1}^{2}}\right) \\
& =C_{2}^{1}-C_{2}^{2} C_{2}^{3},
\end{aligned}
$$

where

$$
\begin{aligned}
C_{2}^{1} & =\mathbf{E}\left[e^{(1-\gamma)\left(\mu_{\rho} y_{1}+\frac{1}{2} \sigma_{\rho}^{2} y_{1}^{2}\right)}\right] \\
& =\frac{1}{\sqrt{1-(1-\gamma) \sigma_{\rho}^{2} \sigma^{2}}} e^{-\frac{1}{2} \frac{(1-\gamma)^{2} \mu_{\rho}^{2} \sigma^{2}+\left(2(1-\gamma) \mu_{\rho}+(1-\gamma) \sigma_{\rho}^{2} \mu\right) \mu}{(1-\gamma) \sigma_{\rho}^{2} \sigma^{2}-1}}, \\
C_{2}^{2} & =\mathbf{E}\left[e^{-\gamma\left(\mu_{\rho} y_{1}+\frac{1}{2} \sigma_{\rho}^{2} y_{1}^{2}\right)}\right] \\
& =\frac{1}{\sqrt{1+\gamma \sigma_{\rho}^{2} \sigma^{2}}} e^{-\frac{1}{2} \frac{\gamma^{2} \mu_{\rho}^{2} \sigma^{2}+\left(-2 \gamma \mu_{\rho}-\gamma \sigma_{\rho}^{2} \mu\right) \mu}{-\gamma \sigma_{\rho}^{2} \sigma^{2}-1}}, \\
C_{2}^{3} & =\mathbf{E}\left[e^{\mu_{\rho} y_{1}+\frac{1}{2} \sigma_{\rho}^{2} y_{1}^{2}}\right] \\
& =\frac{1}{\sqrt{1-\sigma_{\rho}^{2} \sigma^{2}}} e^{-\frac{1}{2} \frac{\mu_{\rho}^{2} \sigma^{2}+\left(2 \mu_{\rho}+\sigma_{\rho}^{2} \mu\right) \mu}{\sigma_{\rho}^{2} \sigma^{2}-1}} .
\end{aligned}
$$


The numerator of the second summand is

$$
\begin{aligned}
\operatorname{Cov}\left(D_{1}^{\rho}, D_{1}^{M}\right) & =\operatorname{Cov}\left(e^{\rho y_{1}}, e^{\mu_{\rho} y_{1}+\frac{1}{2} \sigma_{\rho}^{2} y_{1}^{2}}\right) \\
& =\mathbf{E}\left[e^{\left(\rho+\mu_{\rho}\right) y_{1}+\frac{1}{2} \sigma_{\rho}^{2} y_{1}^{2}}\right]-\mathbf{E}\left[e^{\rho y_{1}}\right] \mathbf{E}\left[e^{\mu_{\rho} y_{1}+\frac{1}{2} \sigma_{\rho}^{2} y_{1}^{2}}\right] \\
& =C_{3}\left(e^{\frac{1}{2} \frac{\left(\rho^{2}+2 \rho \mu_{\rho}\right) \sigma^{2}+2 \rho \mu}{1-\sigma_{\rho}^{2} \sigma^{2}}}-e^{\frac{1}{2}\left(\rho^{2} \sigma^{2}+2 \rho \mu\right)}\right)
\end{aligned}
$$

where

$$
C_{3}=\frac{1}{\sqrt{1-\sigma_{\rho}^{2} \sigma^{2}}} e^{\frac{1}{2} \frac{\mu_{\rho}^{2} \sigma^{2}+2 \mu_{\rho} \mu+\sigma_{\rho}^{2} \mu^{2}}{1-\sigma_{\rho}^{2} \sigma^{2}}} .
$$

The denominator is given by

$$
\begin{aligned}
\operatorname{Var}\left(D_{1}^{M}\right) & =\operatorname{Var}\left(e^{\mu_{\rho} y_{1}+\frac{1}{2} \sigma_{\rho}^{2} y_{1}^{2}}\right) \\
& =\mathbf{E}\left[e^{2 \mu_{\rho} y_{1}+\sigma_{\rho}^{2} y_{1}^{2}}\right]-\mathbf{E}\left[e^{\mu_{\rho} y_{1}+\frac{1}{2} \sigma_{\rho}^{2} y_{1}^{2}}\right]^{2} \\
& =C_{4}^{1}-C_{4}^{2},
\end{aligned}
$$

where

$$
\begin{aligned}
& C_{4}^{1}=\frac{1}{\sqrt{1-2 \sigma_{\rho}^{2} \sigma^{2}}} e^{-\frac{2 \mu_{\rho}^{2} \sigma^{2}+\left(2 \mu_{\rho}+\sigma_{\rho}^{2} \mu\right) \mu}{2 \sigma_{\rho}^{2} \sigma^{2}-1}} \\
& C_{4}^{2}=\frac{1}{1-\sigma_{\rho}^{2} \sigma^{2}} e^{-\frac{\mu_{\rho}^{2} \sigma^{2}+\left(2 \mu_{\rho}+\sigma_{\rho}^{2} \mu\right) \mu}{\sigma_{\rho}^{2} \sigma^{2}-1}}
\end{aligned}
$$

Combining (B.1), (B.2), (B.3), (B.5) and (B.6) we obtain

$$
A^{\rho}=\frac{q^{M}}{q^{\rho}}\left(A_{1}-A_{2}\right)
$$

where

$$
\begin{aligned}
& A_{1}=\frac{C_{1}}{C_{2}^{1}-C_{2}^{2} C_{2}^{3}}\left(e^{\frac{1}{2} \frac{\left(\rho^{2}-2 \rho \gamma \mu \rho\right) \sigma^{2}+2 \rho \mu}{\gamma \sigma_{\rho}^{2} \sigma^{2}+1}}-e^{\frac{1}{2}\left(\rho^{2} \sigma^{2}+2 \rho \mu\right)}\right), \\
& A_{2}=\frac{C_{3}}{C_{4}^{1}-C_{4}^{2}}\left(e^{\frac{1}{2} \frac{\left(\rho^{2}+2 \rho \mu \rho\right) \sigma^{2}+2 \rho \mu}{1-\sigma_{\rho}^{2} \sigma^{2}}}-e^{\frac{1}{2}\left(\rho^{2} \sigma^{2}+2 \rho \mu\right)}\right) .
\end{aligned}
$$


In a next step let us calculate prices. The ratio of the market price over the price of the risky asset is

$$
\begin{aligned}
\frac{q^{M}}{q^{\rho}} & =\frac{\mathbf{E}\left[\delta\left(\frac{c_{0}}{c_{1}}\right)^{\gamma} e^{\mu_{\rho} y_{1}+\frac{1}{2} \sigma_{\rho}^{2} y_{1}^{2}}\right]}{\mathbf{E}\left[\delta\left(\frac{c_{0}}{c_{1}}\right)^{\gamma} e^{\rho y_{1}}\right]}=\frac{\mathbf{E}\left[e^{(1-\gamma)\left(\mu_{\rho} y_{1}+\frac{1}{2} \sigma_{\rho}^{2} y_{1}^{2}\right)}\right]}{\mathbf{E}\left[e^{\left(\rho-\gamma \mu_{\rho}\right) y_{1}-\frac{1}{2} \gamma \sigma_{\rho}^{2} y_{1}^{2}}\right]} \\
& =\frac{\sqrt{1+\gamma \sigma_{\rho}^{2} \sigma^{2}}}{\sqrt{1-(1-\gamma) \sigma_{\rho}^{2} \sigma^{2}}} \frac{e^{-\frac{1}{2} \frac{(1-\gamma)^{2} \mu_{\rho}^{2} \sigma^{2}+2\left((1-\gamma) \mu_{\rho}+\frac{1}{2}(1-\gamma) \sigma_{\rho}^{2} \mu\right) \mu}{(1-\gamma) \sigma_{\rho}^{2} \sigma^{2}-1}}}{e^{-\frac{1}{2} \frac{\left(\rho-\gamma \mu_{\rho}\right)^{2} \sigma^{2}+2\left(\rho-\gamma \mu_{\rho}-\frac{1}{2} \gamma \sigma_{\rho}^{2} \mu\right) \mu}{-\gamma \sigma_{\rho}^{2} \sigma^{2}-1}}} .
\end{aligned}
$$

Plugging this ratio of prices into (B.7), we obtain

$$
A^{\rho}=\frac{\sqrt{1+\gamma \sigma_{\rho}^{2} \sigma^{2}}}{\sqrt{1-(1-\gamma) \sigma_{\rho}^{2} \sigma^{2}}} \frac{e^{-\frac{1}{2} \frac{(1-\gamma)^{2} \mu_{\rho}^{2} \sigma^{2}+2\left((1-\gamma) \mu_{\rho}+\frac{1}{2}(1-\gamma) \sigma_{\rho}^{2} \mu\right) \mu}{(1-\gamma) \sigma_{\rho}^{2} \sigma^{2}-1}}}{e^{-\frac{1}{2} \frac{\left(\rho-\gamma \mu_{\rho}\right)^{2} \sigma^{2}+2\left(\rho-\gamma \mu_{\rho}-\frac{1}{2} \gamma \sigma_{\rho}^{2} \mu\right) \mu}{-\gamma \sigma_{\rho}^{2} \sigma^{2}-1}}}\left(A_{1}-A_{2}\right)
$$

where

$$
\begin{aligned}
& A_{1}=\frac{C_{1}}{C_{2}^{1}-C_{2}^{2} C_{2}^{3}}\left(e^{\frac{1}{2} \frac{\left(\rho^{2}-2 \rho \gamma \mu \rho\right) \sigma^{2}+2 \rho \mu}{\gamma \sigma_{\rho}^{2} \sigma^{2}+1}}-e^{\frac{1}{2}\left(\rho^{2} \sigma^{2}+2 \rho \mu\right)}\right), \\
& A_{2}=\frac{C_{3}}{C_{4}^{1}-C_{4}^{2}}\left(e^{\frac{1}{2} \frac{\left(\rho^{2}+2 \rho \mu \rho\right) \sigma^{2}+2 \rho \mu}{1-\sigma_{\rho}^{2} \sigma^{2}}}-e^{\frac{1}{2}\left(\rho^{2} \sigma^{2}+2 \rho \mu\right)}\right) .
\end{aligned}
$$

Note that $C_{1}, C_{2}^{1}, C_{2}^{2}, C_{2}^{3}, C_{3}, C_{4}^{1}$ and $C_{4}^{2}$ do not depend on $\rho$.

Note that the second summand in (B.1) represents $\beta^{\rho}$. Therefore we have

$$
\beta^{\rho}=\frac{\sqrt{1+\gamma \sigma_{\rho}^{2} \sigma^{2}}}{\sqrt{1-(1-\gamma) \sigma_{\rho}^{2} \sigma^{2}}} \frac{e^{-\frac{1}{2} \frac{(1-\gamma)^{2} \mu_{\rho}^{2} \sigma^{2}+2\left((1-\gamma) \mu_{\rho}+\frac{1}{2}(1-\gamma) \sigma_{\rho}^{2} \mu\right) \mu}{(1-\gamma) \sigma_{\rho}^{2} \sigma^{2}-1}}}{e^{-\frac{1}{2} \frac{(\rho-\gamma \mu \rho)^{2} \sigma^{2}+2\left(\rho-\gamma \mu_{\rho}-\frac{1}{2} \gamma \sigma_{\rho}^{2} \mu\right) \mu}{-\gamma \sigma_{\rho}^{2} \sigma^{2}-1}}} A_{2} .
$$




\section{Appendix C. Derivation of expected returns}

The price of the market portfolio is given by

$$
\begin{aligned}
q^{M} & =\mathbf{E}\left[\delta\left(\frac{c_{0}}{c_{1}}\right)^{\gamma} D_{1}^{M}\right] \\
& =\delta c_{0}^{\gamma} \mathbf{E}\left[e^{(1-\gamma)\left(\mu_{\rho} y_{1}+\frac{1}{2} \sigma_{\rho}^{2} y_{1}^{2}\right)}\right] \\
& =\delta c_{0}^{\gamma} \frac{1}{\sqrt{2 \pi \sigma^{2}}} \int_{-\infty}^{\infty} e^{(1-\gamma)\left(\mu_{\rho} y+\frac{1}{2} \sigma_{\rho}^{2} y^{2}\right)} e^{-\frac{(y-\mu)^{2}}{2 \sigma^{2}}} d y \\
& =\delta c_{0}^{\gamma} \frac{1}{\sqrt{2 \pi \sigma^{2}}} \int_{-\infty}^{\infty} e^{\frac{1}{2}\left((1-\gamma) \sigma_{\rho}^{2}-\frac{1}{\sigma^{2}}\right) y^{2}+\left((1-\gamma) \mu_{\rho}+\frac{\mu}{\sigma^{2}}\right) y-\frac{\mu^{2}}{2 \sigma^{2}}} d y \\
& =\delta c_{0}^{\gamma} \frac{1}{\sqrt{2 \pi \sigma^{2}}} e^{\frac{1}{2} \Sigma_{\gamma}\left((1-\gamma) \mu_{\rho}+\frac{\mu}{\sigma^{2}}\right)^{2}-\frac{\mu^{2}}{2 \sigma^{2}}} \int_{-\infty}^{\infty} e^{-\frac{1}{2 \Sigma_{\gamma}}\left(y-\Sigma_{\gamma}\left((1-\gamma) \mu_{\rho}+\frac{\mu}{\sigma^{2}}\right)\right)^{2}} d y,
\end{aligned}
$$

where

$$
\Sigma_{\gamma}=\frac{\sigma^{2}}{1-(1-\gamma) \sigma^{2} \sigma_{\rho}^{2}} .
$$

Noting that the integral over the density function is 1 , we obtain

$$
q^{M}=\delta c_{0}^{\gamma} \sqrt{\frac{\Sigma_{\gamma}}{\sigma^{2}}} e^{\frac{1}{2} \Sigma_{\gamma}\left((1-\gamma) \mu_{\rho}+\frac{\mu}{\sigma^{2}}\right)^{2}-\frac{\mu^{2}}{2 \sigma^{2}}} .
$$

For the expected future payoff, we have

$$
\mathbf{E}\left[D_{1}^{M}\right]=\mathbf{E}\left[e^{\mu_{\rho} y_{1}+\frac{1}{2} \sigma_{\rho}^{2} y_{1}^{2}}\right] .
$$

Note that the expectation equals the expectation in prices $q^{M}$ for $\gamma=0$. Thus, we have

$$
\mathbf{E}\left[D_{1}^{M}\right]=\sqrt{\frac{\Sigma_{0}}{\sigma^{2}}} e^{\frac{1}{2} \Sigma_{0}\left(\mu_{\rho}+\frac{\mu}{\sigma^{2}}\right)^{2}-\frac{\mu^{2}}{2 \sigma^{2}}} .
$$

Therefore the expected market return is given as

$$
\begin{aligned}
\mathbf{E}\left[R^{M}\right] & =\mathbf{E}\left[\frac{D_{1}^{M}}{q^{M}}\right]=\frac{\sqrt{\frac{\Sigma_{0}}{\sigma^{2}}} e^{\frac{1}{2} \Sigma_{0}\left(\mu_{\rho}+\frac{\mu}{\sigma^{2}}\right)^{2}-\frac{\mu^{2}}{2 \sigma^{2}}}}{\delta c_{0}^{\gamma} \sqrt{\frac{\Sigma_{\gamma}}{\sigma^{2}}} e^{\frac{1}{2} \Sigma_{\gamma}\left((1-\gamma) \mu_{\rho}+\frac{\mu}{\sigma^{2}}\right)^{2}-\frac{\mu^{2}}{2 \sigma^{2}}}} \\
& =\frac{1}{\delta c_{0}^{\gamma}} \sqrt{\frac{\Sigma_{0}}{\Sigma_{\gamma}}} e^{\frac{1}{2} \Sigma_{0}\left(\mu_{\rho}+\frac{\mu}{\sigma^{2}}\right)^{2}-\frac{1}{2} \Sigma_{\gamma}\left((1-\gamma) \mu_{\rho}+\frac{\mu}{\sigma^{2}}\right)^{2}} .
\end{aligned}
$$


For the riskless asset, we have

$$
\begin{aligned}
q^{f} & =\mathbf{E}\left[\delta\left(\frac{c_{0}}{c_{1}}\right)^{\gamma}\right] \\
& =\delta c_{0}^{\gamma} \mathbf{E}\left[e^{-\gamma\left(\mu_{\rho} y_{1}+\frac{1}{2} \sigma_{\rho}^{2} y_{1}^{2}\right)}\right] .
\end{aligned}
$$

Note that the expectation equals the expectation in prices $q^{M}$ if we substitute $\gamma$ by $1+\gamma$. Therefore we have

$$
q^{f}=\delta c_{0}^{\gamma} \sqrt{\frac{\Sigma_{\gamma+1}}{\sigma^{2}}} e^{\frac{1}{2} \Sigma_{\gamma+1}\left(-\gamma \mu_{\rho}+\frac{\mu}{\sigma^{2}}\right)^{2}-\frac{\mu^{2}}{2 \sigma^{2}}} .
$$

There riskless rate is

$$
R^{f}=\frac{1}{\delta c_{0}^{\gamma}} \sqrt{\frac{\sigma^{2}}{\Sigma_{\gamma+1}}} e^{-\frac{1}{2} \Sigma_{\gamma+1}\left(-\gamma \mu_{\rho}+\frac{\mu}{\sigma^{2}}\right)^{2}+\frac{\mu^{2}}{2 \sigma^{2}}}
$$

\section{Appendix D. Properties of the function $A^{\rho}$ when $D^{M}$ is lognormal}

In order to determine the shape of the function, we compute possible extrema. Recall that

$$
A^{\rho}=\frac{e^{-\gamma \sigma^{2}}}{e^{-\rho \gamma \sigma^{2}}}\left(\frac{e^{-\rho \gamma \sigma^{2}}-1}{e^{-\gamma \sigma^{2}}-1}-\frac{e^{\rho \sigma^{2}}-1}{e^{\sigma^{2}}-1}\right) .
$$

Taking the first derivative with respect to $\rho$ and setting it equal to zero, we obtain

$$
-\frac{\gamma \sigma^{2} e^{\rho \gamma \sigma^{2}}}{e^{-\gamma \sigma^{2}}-1}-\frac{\sigma^{2}(\gamma+1) e^{\rho \sigma^{2}(\gamma+1)}}{e^{\sigma^{2}}-1}+\frac{\gamma \sigma^{2} e^{\rho \gamma \sigma^{2}}}{e^{\sigma^{2}}-1}=0 .
$$

This is equivalent to

$$
\gamma \sigma^{2}\left(e^{-\gamma \sigma^{2}}-e^{\sigma^{2}}\right) e^{\rho \gamma \sigma^{2}}-\sigma^{2}(\gamma+1)\left(e^{-\gamma \sigma^{2}}-1\right) e^{\rho \sigma^{2}(\gamma+1)}=0 .
$$

Substituting $x=e^{\rho \sigma^{2}}$, we obtain the polynomial

$$
\left(\gamma \sigma^{2}\left(e^{-\gamma \sigma^{2}}-e^{\sigma^{2}}\right)-\sigma^{2}(\gamma+1)\left(e^{-\gamma \sigma^{2}}-1\right) x\right) x^{\gamma}=0 .
$$

The two solutions to this equation are

$$
\begin{aligned}
& x=0, \\
& x=\frac{\gamma\left(e^{-\gamma \sigma^{2}}-e^{\sigma^{2}}\right)}{(\gamma+1)\left(e^{-\gamma \sigma^{2}}-1\right)} .
\end{aligned}
$$


Note that $x=0$ only occurs for $\rho=-\infty$. Thus, the extremum of interest is only

$$
\rho=\frac{1}{\sigma^{2}} \ln \left(\frac{\gamma\left(e^{-\gamma \sigma^{2}}-e^{\sigma^{2}}\right)}{(\gamma+1)\left(e^{-\gamma \sigma^{2}}-1\right)}\right) .
$$

Next we want to show that it is a maximum. Thus, we verify if the second derivative is negative at the extremum. We want to show that

$$
-\frac{\gamma^{2} \sigma^{4} e^{\rho \gamma \sigma^{2}}}{e^{-\gamma \sigma^{2}}-1}-\frac{\sigma^{4}(\gamma+1)^{2} e^{\rho \sigma^{2}(\gamma+1)}}{e^{\sigma^{2}}-1}+\frac{\gamma^{2} \sigma^{4} e^{\rho \gamma \sigma^{2}}}{e^{\sigma^{2}}-1}<0 .
$$

In terms of $x$, we have

$$
\left(\frac{\gamma^{2} \sigma^{4}}{e^{\sigma^{2}}-1}-\frac{\gamma^{2} \sigma^{4}}{e^{-\gamma \sigma^{2}}-1}-\frac{\sigma^{4}(\gamma+1)^{2}}{e^{\sigma^{2}}-1} x\right) x^{\gamma}<0 .
$$

Note that $x$ and thus $x^{\gamma}$ are positive, since the numerator as well as the denominator is negative at the extremum. Therefore we only have to verify that

$$
\frac{\gamma^{2} \sigma^{4}}{e^{\sigma^{2}}-1}-\frac{\gamma^{2} \sigma^{4}}{e^{-\gamma \sigma^{2}}-1}-\frac{\sigma^{4}(\gamma+1)^{2}}{e^{\sigma^{2}}-1} x<0
$$

Inserting $x$ at the extremum yields

$$
\frac{\gamma^{2} \sigma^{4}}{e^{\sigma^{2}}-1}-\frac{\gamma^{2} \sigma^{4}}{e^{-\gamma \sigma^{2}}-1}-\frac{\sigma^{4}(\gamma+1)^{2}}{e^{\sigma^{2}}-1} \frac{\gamma\left(e^{-\gamma \sigma^{2}}-e^{\sigma^{2}}\right)}{(\gamma+1)\left(e^{-\gamma \sigma^{2}}-1\right)}<0 .
$$

Multiplying by $\left(e^{\sigma^{2}}-1\right)(\gamma+1)\left(e^{-\gamma \sigma^{2}}-1\right)$, we get

$$
(\gamma+1) \gamma^{2} \sigma^{4}\left(e^{-\gamma \sigma^{2}}-1\right)-(\gamma+1) \gamma^{2} \sigma^{4}\left(e^{\sigma^{2}}-1\right)-(\gamma+1)^{2} \gamma \sigma^{4}\left(e^{-\gamma \sigma^{2}}-e^{\sigma^{2}}\right)>0 .
$$

Removing the canceling terms we have

$$
e^{\sigma^{2}}-e^{-\gamma \sigma^{2}}>0
$$

which is true. Therefore the function $A^{\rho}$ has only one extremum for finite $\rho$, which is a maximum. 


\section{Appendix E. Properties of the function $\beta^{\rho}$ when $D^{M}$ is lognormal}

Now let us turn to the CAPM beta $\beta^{\rho}$ as a function of $\rho$. Recall that

$$
\beta^{\rho}=\frac{e^{-\gamma \sigma^{2}}\left(e^{\rho \sigma^{2}}-1\right)}{e^{-\rho \gamma \sigma^{2}}\left(e^{\sigma^{2}}-1\right)} .
$$

Setting the first derivative to zero, we obtain

$$
\frac{e^{-\gamma \sigma^{2}}}{e^{\sigma^{2}}-1}\left((\gamma+1) \sigma^{2} e^{\rho \sigma^{2}(\gamma+1)}-\gamma \sigma^{2} e^{\rho \gamma \sigma^{2}}\right)=0 .
$$

This equation is satisfied by $\rho=-\infty$ and $\rho=\frac{1}{\sigma^{2}} \ln \left(\frac{\gamma}{\gamma+1}\right)$. In the following, we show that the finite extremum is a minimum and the second derivative is

$$
\frac{e^{-\gamma \sigma^{2}}}{e^{\sigma^{2}}-1}\left((\gamma+1)^{2} \sigma^{4} e^{\rho \sigma^{2}(\gamma+1)}-\gamma^{2} \sigma^{4} e^{\rho \gamma \sigma^{2}}\right)>0
$$

This is equivalent to

$$
(\gamma+1)^{2} e^{\rho \sigma^{2}}-\gamma^{2}>0
$$

which is true. Since $A^{\rho}$ is decreasing in $\rho$ for values larger than

$$
\rho=\frac{1}{\sigma^{2}} \ln \left(\frac{\gamma\left(e^{-\gamma \sigma^{2}}-e^{\sigma^{2}}\right)}{(\gamma+1)\left(e^{-\gamma \sigma^{2}}-1\right)}\right)
$$

and $\beta^{\rho}$ is increasing in $\rho$ for values larger than $\rho=\frac{1}{\sigma^{2}} \ln \left(\frac{\gamma}{\gamma+1}\right)$, we see that $A^{\rho}$ has to decrease in $\beta^{\rho}$ for values of $\rho$ larger than

$$
\rho=\frac{1}{\sigma^{2}} \ln \left(\frac{\gamma\left(e^{-\gamma \sigma^{2}}-e^{\sigma^{2}}\right)}{(\gamma+1)\left(e^{-\gamma \sigma^{2}}-1\right)}\right) .
$$

\section{Appendix F. Plots of $A^{\rho}$ and $\beta^{\rho}$ when $D^{M}$ is lognormal}

Figure F.6 shows the factor $A^{\rho}$ and the CAPM beta $\beta^{\rho}$ as a function of $\rho$. Recall that the pricing error is given by

$$
\alpha^{\rho}=A^{\rho}\left(\mathbf{E}\left[R^{M}\right]-R^{f}\right) .
$$

$A^{\rho}$ therefore describes the pricing error as a proportion of the market equity premium. We see that the pricing error can become a substantial fraction of the equity premium for a certain range of assets and higher coefficients of relative risk aversion. 

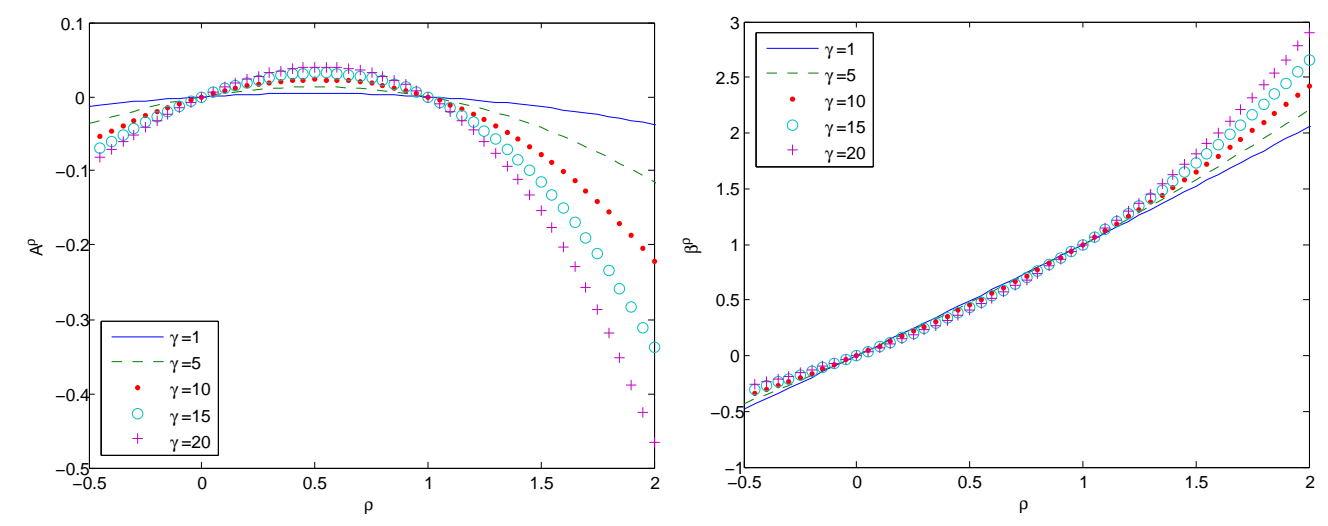

Figure F.6: The left plot shows the factor $A^{\rho}$ of the pricing error as a function of $\rho$ for different coefficients of relative risk aversion. The right plot shows the CAPM beta as a function of $\rho$ for different coefficients of relative risk aversion. The standard deviation of logarithmic dividend growth is set to $13.5 \%$.

\section{Appendix G. The shape of $q^{\rho}$ when $D^{M}$ is lognormal}

The price of an asset is

$$
q^{\rho}=\mathbf{E}\left[\delta\left(\frac{c_{0}}{c_{1}}\right)^{\gamma} e^{\rho y}\right]=\delta c_{0}^{\gamma} e^{(\rho-\gamma) \mu+\frac{1}{2}(\rho-\gamma)^{2} \sigma^{2}}
$$

In order to determine local extrema, we take the first order condition and set it to zero:

$$
\delta c_{0}^{\gamma}\left(\mu+(\rho-\gamma) \sigma^{2}\right) e^{(\rho-\gamma) \mu+\frac{1}{2}(\rho-\gamma)^{2} \sigma^{2}}=0 .
$$

Thus, there is an extremum for

$$
\rho=\gamma-\frac{\mu}{\sigma^{2}}
$$

In order to determine if this is a maximum or a minimum, we compute the second derivative

$$
\delta c_{0}^{\gamma}\left(\sigma^{2}+\left(\mu+(\rho-\gamma) \sigma^{2}\right)^{2}\right) e^{(\rho-\gamma) \mu+\frac{1}{2}(\rho-\gamma)^{2} \sigma^{2}}>0
$$

The function therefore has only one minimum. 


\section{Appendix H. First two moments of logarithmic consumption growth}

The first moment of logarithmic consumption growth is

$$
\mathbf{E}\left[\ln \left(\frac{c_{1}}{c_{0}}\right)\right]=\mathbf{E}\left[\ln \left(c_{1}\right)\right]-\ln \left(c_{0}\right)
$$

and we have

$$
\mathbf{E}\left[\ln \left(c_{1}\right)\right]=\mathbf{E}\left[\mu_{\rho} y_{1}+\frac{1}{2} \sigma_{\rho}^{2} y_{1}^{2}\right]=\mu_{\rho} \mathbf{E}\left[y_{1}\right]+\frac{1}{2} \sigma_{\rho}^{2} \mathbf{E}\left[y_{1}^{2}\right]=\mu_{\rho} \mu+\frac{1}{2} \sigma_{\rho}^{2}\left(\mu^{2}+\sigma^{2}\right) .
$$

The second moment of logarithmic consumption growth is

$$
\operatorname{Var}\left(\ln \left(\frac{c_{1}}{c_{0}}\right)\right)=\operatorname{Var}\left(\ln \left(c_{1}\right)\right)
$$

and we have

$$
\begin{aligned}
\operatorname{Var}\left(\ln \left(c_{1}\right)\right)= & \operatorname{Var}\left(\mu_{\rho} y_{1}+\frac{1}{2} \sigma_{\rho}^{2} y_{1}^{2}\right) \\
= & \mathbf{E}\left[\left(\mu_{\rho} y_{1}+\frac{1}{2} \sigma_{\rho}^{2} y_{1}^{2}\right)^{2}\right]-\left(\mu_{\rho} \mu+\frac{1}{2} \sigma_{\rho}^{2}\left(\mu^{2}+\sigma^{2}\right)\right)^{2} \\
= & \mathbf{E}\left[\mu_{\rho}^{2} y_{1}^{2}+\mu_{\rho} \sigma_{\rho}^{2} y_{1}^{3}+\frac{1}{4} \sigma_{\rho}^{4} y_{1}^{4}\right] \\
& -\left(\mu_{\rho}^{2} \mu^{2}+\mu_{\rho} \mu \sigma_{\rho}^{2}\left(\mu^{2}+\sigma^{2}\right)+\frac{1}{4} \sigma_{\rho}^{4}\left(\mu^{2}+\sigma^{2}\right)^{2}\right) \\
= & \mu_{\rho}^{2}\left(\mu^{2}+\sigma^{2}\right)+\mu_{\rho} \sigma_{\rho}^{2}\left(\mu^{3}+3 \mu \sigma^{2}\right)+\frac{1}{4} \sigma_{\rho}^{4}\left(\mu^{4}+6 \mu^{2} \sigma^{2}+3 \sigma^{4}\right) \\
& -\mu_{\rho}^{2} \mu^{2}-\mu_{\rho} \sigma_{\rho}^{2} \mu^{3}-\mu_{\rho} \sigma_{\rho}^{2} \mu \sigma^{2}-\frac{1}{4} \sigma_{\rho}^{4} \mu^{4}-\frac{1}{2} \sigma_{\rho}^{4} \mu^{2} \sigma^{2}-\frac{1}{4} \sigma_{\rho}^{4} \sigma^{4} \\
= & \mu_{\rho}^{2} \sigma^{2}+2 \mu_{\rho} \sigma_{\rho}^{2} \mu \sigma^{2}+\sigma_{\rho}^{4} \mu^{2} \sigma^{2}+\frac{1}{2} \sigma_{\rho}^{4} \sigma^{4} .
\end{aligned}
$$

Matching the first moment (H.1), we obtain

$$
\mu_{\rho} \mu+\frac{1}{2} \sigma_{\rho}^{2}\left(\mu^{2}+\sigma^{2}\right)=0.067+\ln (10000) .
$$


Matching the second moment (H.2), we obtain

$$
\mu_{\rho}^{2} \sigma^{2}+2 \mu_{\rho} \sigma_{\rho}^{2} \mu \sigma^{2}+\sigma_{\rho}^{4} \mu^{2} \sigma^{2}+\frac{1}{2} \sigma_{\rho}^{4} \sigma^{4}=0.135^{2} .
$$

Combining (H.3) and (H.4), we obtain

$$
\frac{1}{2} \sigma_{\rho}^{4} \sigma^{4}-\left(\mu_{\rho}^{2}+2 \sigma_{\rho}^{2}(0.067+\ln (10000))\right) \sigma^{2}+0.135^{2}=0 .
$$

The solutions to this equation are

$$
\sigma^{2}=\frac{\mu_{\rho}^{2}+2 \sigma_{\rho}^{2}(0.067+\ln (10000)) \pm \sqrt{\left(\mu_{\rho}^{2}+2 \sigma_{\rho}^{2}(0.067+\ln (10000))\right)^{2}-2 \sigma_{\rho}^{4} \cdot 0.135^{2}}}{\sigma_{\rho}^{4}} .
$$

Solving (H.3) for $\mu$, we obtain

$$
\mu=\frac{-\mu_{\rho} \pm \sqrt{\mu_{\rho}^{2}-\sigma_{\rho}^{2}\left(\sigma_{\rho}^{2} \sigma^{2}-2(0.067+\ln (10000))\right.}}{\sigma_{\rho}^{2}} .
$$

Only two of the solutions are real. We take the one that gives positive parameter values. 


\section{References}

Banz, R. W., 1981. The relationship between return and market value of common stocks. Journal of Financial Economics 9 (1), 3-18.

Barro, R. J., 2006. Rare disasters and asset markets in the twentieth century. The Quarterly Journal of Economics 121 (3), 823-866.

Berk, J. B., 1997. Necessary conditions for the CAPM. Journal of Economic Theory 73 (1), 245-257.

Black, F., Jensen, M. C., Scholes, M., 1972. The capital asset pricing model: some empirical tests. In: Jensen, M. C. (Ed.), Studies in the Theory of Capital Markets. Praeger Publishers Inc., New York, pp. 79-121.

Breeden, D. T., 1979. An intertemporal asset pricing model with stochastic consumption and investment opportunities. Journal of Financial Economics 7 (3), 265-296.

Caliskan, N., Hens, T., 2013. Value around the world. Working paper.

Campbell, J., Viceira, L., 2002. Strategic Asset Allocation: Portfolio Choice for Long-Term Investors. Oxford University Press, Oxford.

Chen, L., 2009. On the reversal of return and dividend growth predictability: A tale of two periods. Journal of Financial Economics 92 (1), 128-151.

Dittmar, R. F., 2002. Nonlinear pricing kernels, kurtosis preference, and evidence from the cross section of equity returns. Journal of Finance 57 (1), 369-403.

Elmiger, S., 2010. Market selection in an evolutionary market with dividends generated by a percolation model. Master's thesis.

Fama, E. F., French, K. R., 1993. Common risk factors in the returns on stocks and bonds. Journal of Financial Economics 33 (1), 3-56.

Fama, E. F., French, K. R., 2004. The capital asset pricing model: theory and evidence. The Journal of Economic Perspectives 18 (3), 25-46.

Frazzini, A., Pedersen, L. H., 2011. Betting against beta. Working paper. 
Harvey, C. R., Siddique, A., 2000. Conditional skewness in asset pricing tests. Journal of Finance 5 (3), 1263-1295.

Kraus, A., Litzenberger, R., 1983. On the distributional conditions for a consumption-oriented three moment CAPM. Journal of Finance 38 (5), $1381-1391$.

LeRoy, S. F., Werner, J., 2001. Principles of Financial Economics. Cambridge University Press, Cambridge.

Lintner, J., 1965. The valuation of risk assets and the selection of risky investments in stock portfolios and capital budgets. Review of Economics and Statistics 47 (1), 13-37.

Lucas, R. E., 1978. Asset prices in an exchange economy. Econometrica 46 (6), 1429-1445.

Merton, R. C., 1973. An intertemporal capital asset pricing model. Econometrica 41 (5), 867-887.

Mossin, J., 1966. Equilibrium in a capital asset market. Econometrica 34 (4), 768-783.

Rietz, T. A., 1988. The equity risk premium: A solution. Journal of Monetary Economics 22 (1), 117-131.

Rosenberg, B., Reid, K., Lanstein, R., 1985. Persuasive evidence of market inefficiency. Journal of Portfolio Management 11 (3), 9-16.

Rubinstein, M., 1976. The valuation of uncertain income streams and the pricing of options. The Bell Journal of Economics 7 (2), 407-425.

Sharpe, W. F., 1964. Capital asset prices: A theory of market equilibrium under conditions of risk. Journal of Finance 19 (3), 425-442. 


\section{swiss : finance: institute}

c/o University of Geneva

40 bd du Pont d'Arve

1211 Geneva 4

Switzerland

$\mathrm{T}+41223798471$

F +41 223798277

RPS@sfi.ch

www.SwissFinanceInstitute.ch 